\title{
DIFFUSION TENSOR IMAGING OF THE INTERNAL CAPSULE AND CORPUS CALLOSUM OF PRETERM INFANTS BORN UNDER 28 WEEKS GESTATION
}

\author{
J.J. Atkinson ${ }^{1}$, L.M. Parkes ${ }^{2,3}$, C. Gamble ${ }^{4}$, S.M. Ng ${ }^{5}$, M. Turner ${ }^{5}$, A.M. Weindling ${ }^{5}$, L.J. Abernethy ${ }^{5,6}$, V. \\ Sluming ${ }^{1}$ \\ ${ }^{I}$ Department of Molecular and Cellular Biology, Institute of Translational Medicine, University of Liverpool, \\ Liverpool, ${ }^{2}$ Imaging Sciences and Biomedical Engineering, School of Cancer and Enabling Sciences, \\ ${ }^{3}$ Biomedical Imaging Institute, University of Manchester, Manchester, ${ }^{4}$ Department for Biostatistics, Institute \\ of Translational Medicine, ${ }^{5}$ School of Reproductive and Developmental Medicine, University of Liverpool, \\ ${ }^{6}$ Department of Radiology, Alder Hey Children's Hospital, Liverpool, UK
}

Introduction: Previous research has shown that white matter (WM) structures in the human brain develop at different rates. Development of these structures can be estimated with Diffusion Tensor Imaging (DTI). Higher fractional anisotropy (FA) and lower apparent diffusion coefficient (ADC) are associated with more extensive myelination. Previous studies have not reported the pattern of these parameters in neonates born at extreme prematurity. This study aims to compare FA and ADC between different brain regions at term age in infants born at less than 28 weeks' gestation.

Method: 51 babies (28 male, 23 female) were imaged on a 1.5 Telsa MRI Scanner. DTI analysis was performed using medical imaging software (DTIstudio). FA and ADC maps were used to draw regions of interest around the Internal Capsule (IC), Corpus Callosum (CC), Frontal Lobes (FL) and Occipital Lobes (OL). Statistical analysis was conducted with repeated measures ANOVA using SPSS version 18.

Results: Posterior CC, mean (sd), 0.3760 (0.06749) had significantly ( $\mathrm{p}=0.018)$ higher FA than the Anterior CC 0.3380 (0.06236) on repeated measures ANOVA. Mean (sd) FA was higher in the occipital (Right/Left) $0.1533 / 0.1520(0.03309 / 0.03121)$ than frontal WM $0.1333 / 0.1487(0.03867 / 0.04291)$. This only reached significance $(\mathrm{p}=0.046)$ when comparing mean FA in the right occipital WM to the right frontal WM. ADC values were similar.

Conclusion: These patterns are similar to those of babies born at term. Although previous work has suggested that complications of birth at extreme prematurity disrupt specific aspects of development, these results suggest that the overall developmental programme remains intact. 